\title{
Numerical study of Single-turn and Multi-turn coil effect in the induction heating process
}

\author{
Abdoljabbar Shokri", Hamed Heydari**, Muhammed Jameel M. Asaad ${ }^{* * * * *}$ \\ *Assistant lecturer, Physics department, Payam-e-noor University, Tehran, Iran. \\ ** Physics department, Bu-ali sina University, Hamedan, Iran. \\ ** Assistant lecturer, Physics department, College of education, University of \\ Garmian, Kalar, KRG, Iraq.
}

\section{Abstract}

Induction heating has an important role in the industrial applications. A typical induction heating system has three main parts where they are electrical source, the RF-coil and work piece. The RF-coil is one of the important parameters in setup of induction heating system. In this paper, we have been studied two kinds of coil (Single-turn and Multi-turn) using Finite Element Method (FEM) and by COMSOL MULTIPHYICS package. The results show that considering of each of this coil has significant effect on the electromagnetic fields, eddy currents and generated heat in the coil and work piece.

\section{Keywords: Numerical, induction heating, coil, electromagnetic}

\section{Introduction}

"Induction heating is a method of heating electrically conductive materials taking advantage of the heat produced by the eddy currents generated in the material. It has many advantages compared to other heating systems (e.g. gas and oil-fired furnaces), such as quicker heating, faster start-up, more energy saving and higher production rates" [1]. "The research done these last years in specific power supplies for this application, the numerical and computational methods developed, as well as the decrease of the cost of these systems, has lead to a widespread of Induction heating in many processes and applications, such as cooking, melting, and other magnetic designs"[2,3].

The most basic elements composing an induction heating system are the piece to be heated, also known as a workpiece, and the inductor or coil that produces the magnetic field needed to generate the heat. The inductor and the workpiece can 
have any shape and the piece is usually placed inside the coil to have a better coupling. Considering that this study focuses on the heating of round wires, the inductor used is a solenoid and the workpiece a cylindrical. Inductionheating phenomenon is based on energy losses due to Joule effect. When applying an alternating voltage to an induction coil, an alternating current is generated in the coil. This current produces an alternating magnetic field (Ampere's law) that induces voltage in the workpiece, which opposes to the variation of this magnetic field (Lenz's law). This voltage creates currents in the workpiece, called eddy currents, which have the same frequency but opposite direction than the original current (Fig.1). These eddy currents produce heat in the piece by Joule effect $\left(I^{2} R\right)$ $[1,4]$. Designing of an induction heating system; based on trial-and-error process is much costly, and then the computer modeling is a powerful tool for designing of it.

RF-Coil the one of the critical parts in the induction heating system. For instance, the papers of [5,6] have been studied of cross-section of RF-coil using computer modeling. The aim of this article is study of two types (Single-turn coil and Multi-turn coil) of RF-coil. The Single-turn coil (Fig.2a) is including of turn with cross-section solid while the Multi-turn coil (Fig.2b) is consisting of many tightly-wound conducting wiresseparated by an electrical insulator[7]. We have been computed electromagnetic fields, eddy currents (in the work piece), and generated heat distribution and volumetric in the work piece for both of the coil models.

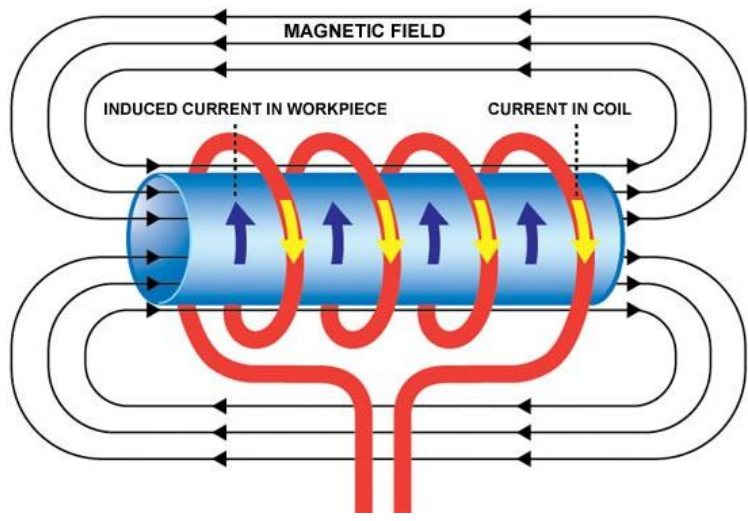

(a)

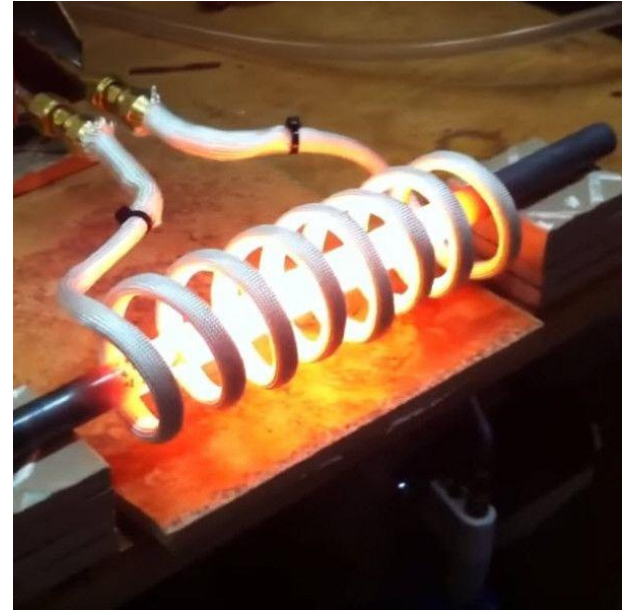

(b)

Figure 6. (a) Schematic induction heating process, (b) real image induction heating process. 


\section{Mathmatical Modeling}

For obtaining of electromagnetic fields the Maxwell's equation should be solved [4-6].It can be summarized as follows. The assumptions are: (i) The system is axisymmetric, (ii) all materials are isotropic, nonmagnetic and have no net electric charge, (iii) the displacement current is neglected, (iv) the distribution of electrical current (also voltage) in the RF-coil is uniform, (v) the currents (impressed and induced) have a steady state quality and as a result the electromagnetic field quantities are harmonically oscillating functions with a single frequency. Under these assumptions, the governing equations are:

$$
\begin{gathered}
\operatorname{curl} \mathbf{E}=-\frac{\partial \mathbf{B}}{\partial t} \\
\operatorname{div} \mathbf{D}=\rho \\
\operatorname{curl} \mathbf{H}=\mathbf{J}_{\text {ext }}+\frac{\partial \mathbf{D}}{\partial t} \\
\operatorname{div} \mathbf{B}=0
\end{gathered}
$$

Where $\mathbf{E}$ is the electric field intensity vector, $\mathbf{B}$ is the magnetic flux density vector, $\mathbf{H}$ is the magnetic field intensity vector, $\mathbf{D}$ is the electric flux density vector, $\mathbf{J}$ is the field current density vector, $\rho$ is the volume charge density and $\mathbf{t}$ is the time.

The following electromagnetic equation is solved for the amplitude of the magnetic vector potential, $\mathbf{A}(\mathrm{Wb} / \mathrm{m})$, with isotropic material properties and an externally-applied sinusoidal-varying current [8]:

$$
\nabla^{2} \mathbf{A}+\omega^{2} \mu \varepsilon \mathbf{A}-i \mu \omega \sigma \mathbf{A}=-\mu \mathbf{J}_{\text {ext }}
$$

Where $\omega$ is angular frequency of the external alternating current $(\mathrm{rad} / \mathrm{s}) ; \mu$ is magnetic permeability (Vs/A m), $\varepsilon$ is the permittivity $(\mathrm{s} / \mathrm{m} \mathrm{ohm}), \sigma$ is the electrical conductivity $(1 / \mathrm{m} \mathrm{ohm}), \mathbf{J}_{e x t}$ the current density vector $\left(\mathrm{A} / \mathrm{m}^{2}\right)$ and in the twodimensional axisymmetric problem is to simply $\mathbf{J}_{e x t}=J_{e x t} \mathbf{e}_{\varphi}$ thus in two-dimensional asymmetric Eq. (5) is to scalar equation:

$$
\frac{1}{r} \frac{\partial}{\partial r}\left(r \frac{\partial A_{\varphi}}{\partial r}\right)+\frac{\partial^{2} A_{\varphi}}{\partial z^{2}}+\omega^{2} \mu \varepsilon A_{\varphi}-i \mu \omega \sigma A_{\varphi}=-\mu J_{e x t \varphi}
$$


With the external current in the coil for high frequency, the second term on the left side of Eq. (6) can be neglected, and the heat source $Q\left(\mathrm{~W} / \mathrm{m}^{3}\right)$ is from resistance heating:

$$
Q\left(W / m^{3}\right)=\frac{\left|\mathbf{J}_{t o t}\right|^{2}}{2 \sigma}
$$

Where the total current density, $\mathbf{J}_{\text {tot }}=\mathbf{J}_{\text {eddy }}+\mathbf{J}_{\text {ext }}$, is the sum of the induced eddy current and the external applied current density, thus:

$$
Q\left(W / m^{3}\right)= \begin{cases}\frac{\left|\mathbf{J}_{e d d y}+\mathbf{J}_{\text {ext }}\right|^{2}}{2 \sigma_{c o}} & \text { in the coil } \\ \frac{\left|\mathbf{J}_{e d d y}\right|^{2}}{2 \sigma_{w}} & \text { in the workpeice }\end{cases}
$$

So, for free space or non-conductivity materials $Q\left(W / \mathrm{m}^{3}\right)=0$.

\section{The calculation Conditions}

For solution of mentioned equations in the previous section, the Finite Element Method (FEM) and COMSOL MULTIPHYSIC software package has been employed. The coil conductors are made by copper tubes of rectangular crosssection with $1 \mathrm{~cm} \times 3 \mathrm{~cm}$ edges, in order to allow the cooling of the inductor by water circulation. The optimum thickness of copper to carry the current has been obtain from relation $d_{o p t}=\delta_{c o i l} \times \pi / 2$, that $d_{o p t}$ thickness and $\delta_{\text {coil }}$ is penetration depth $\left(\delta_{\text {coil }}=503 \sqrt{\rho_{C u} / f}[\mathrm{~m}]\right.$ ) [2,9]. The skin depth of the copper at properties frequency and other parameters used in the calculations has been presented in the Table1. 


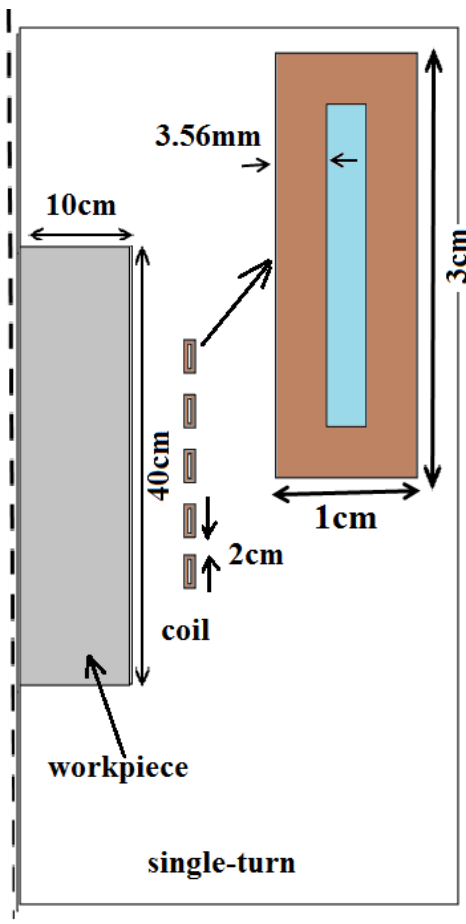

(a)

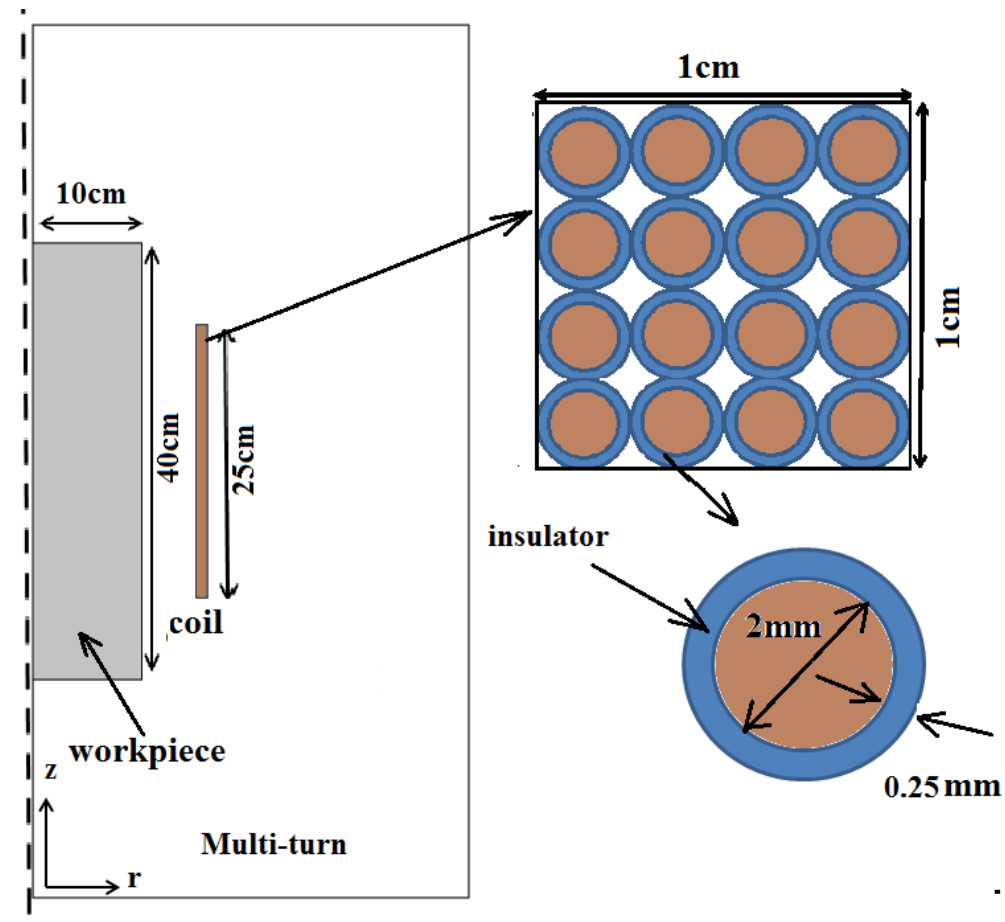

(b)

Figure 2. Induction heating mode COMSOL; (a) Single-turn and (b) Multi-turn.

Table 1. Material Properties for Modeling

\begin{tabular}{lll}
\hline & Symbol & Value \\
\hline Frequency & $f$ & $1000 \mathrm{~Hz}$ \\
\hline Voltage & $V$ & 200 Volt \\
\hline Relative magnetic permeability & $\mu_{r}$ & $\cong 1$ \\
\hline Coil conductivity & $\sigma_{\text {Coil }}$ & $5.9 \times 10^{7}[\mathrm{~s} / \mathrm{m}]$ \\
\hline Workpiece conductivity & $\sigma_{W}$ & $4.0 \times 10^{6}[\mathrm{~s} / \mathrm{m}]$ \\
\hline Skin depth & $d$ & $3.56 \mathrm{~mm}$ \\
\hline Copper resistivity & $\rho_{C u}$ & $1.60 \times 10^{-8}[\Omega . m]$ \\
\hline
\end{tabular}

\section{Result and Discussion}

This section describes the simulations performed with the numerical Finite Element Method (FEM) software COMSOL MULTIPHYSICS for the analysis of the Single-turn and multi-turn coils system [10]. In the Fig.3 the vector magnetic potential distribution for both of two coils (Single-turn and Multi-turn coil) has been shown. According to it, the intensity maximum of vector potential for Single- 
turn coil is approximate $4 \times 10^{-4}[\mathrm{~Wb} / \mathrm{m}]$, where it concentrated at the upper and lowest of coil turns corner also intensity of vector potential has been decreased in the middle turns of coil. The intensity maximum of vector potential for Multi-turn coil is $1 \times 10^{-6}[\mathrm{~Wb} / \mathrm{m}]$, as it has been distributed on the whole of the coil and intensity of vector potential has been decreased in the two ends of coil. The intensity minimum of vector potential for Single-turn coil and Multi-turn coil has been taken place on the middle of workpiece surface. The value maximum of vector potential in the Single-turn coil several times of Multi-turn coil maximum value. Likewise, the vector potential gradient between workpiec and coil in the r-direction in the Multiturn coil faster than Single-turn coil.

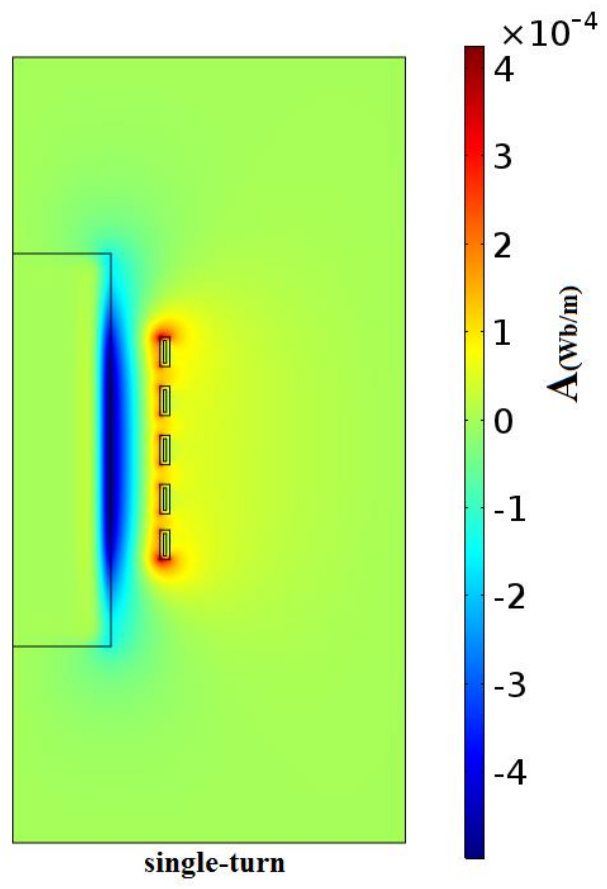

(a)

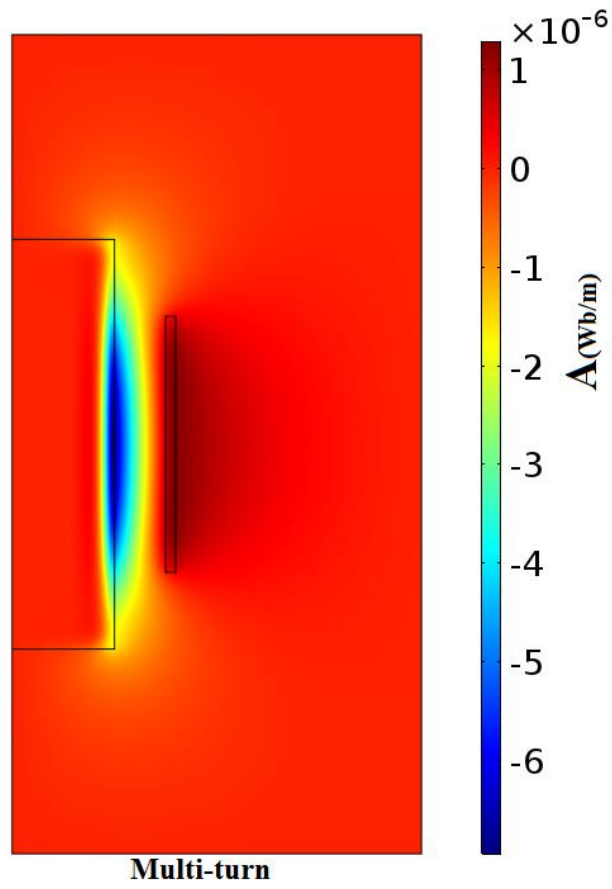

(b)

Figure 3. Intensity and distribution magnetic vector potential; (a) Single-turn coil and(b) Multi-turn coil.

The eddy current density distribution and intensity in the workpiece induction heating system is one of the important parameters for a single-turn and multi-turn investigated. Fig.4 shows that eddy current is concentrated in the layer near the surface of the workpiece and the same phenomenon of skin effect [3] that occurs in the process of induction heating. The maximums value of eddy current in the workpiece for Single-turn coil and Multi-turn coil approximate are $\left(1.4 \times 10^{7}\left[\mathrm{~A} / \mathrm{m}^{2}\right]\right)$ and $\left(2 \times 10^{5}\left[\mathrm{~A} / \mathrm{m}^{2}\right]\right)$, respectively. With attention to Fig.4 can be see that eddy currents density intensity maximum in the single-turn coil is 70 times multi-turn coil. Also the changes of eddy current distribution in the work piece for Single-turn coil is faster than the Multi-turn coil. 
Fig. 5 shows the heat source (power density distribution) generated by the total current inside the conducting regions. Heat is mainly generated in the surface layers (skins) of the induction coil. This power distribution is clearly consistent with the total current density distribution. The value maximum of generated heat in the workpiece for Single-turn coil is approximate $4.5 \times 10^{7}\left[\mathrm{~W} / \mathrm{m}^{3}\right]$, while the for Multiturn coil is $9 \times 10^{3}\left[\mathrm{~W} / \mathrm{m}^{3}\right]$. Comprising these maximum values, we can be found the intensity maximum in the work piece in presence of Single-turn coil 500 times of Multi-turn coil. Also, the pattern of generated heat on the surface of workpiece not same for both of two coils. As one of the induction heating application is hardening of workpiece surface, then the generated heat profile on its surface is critical.

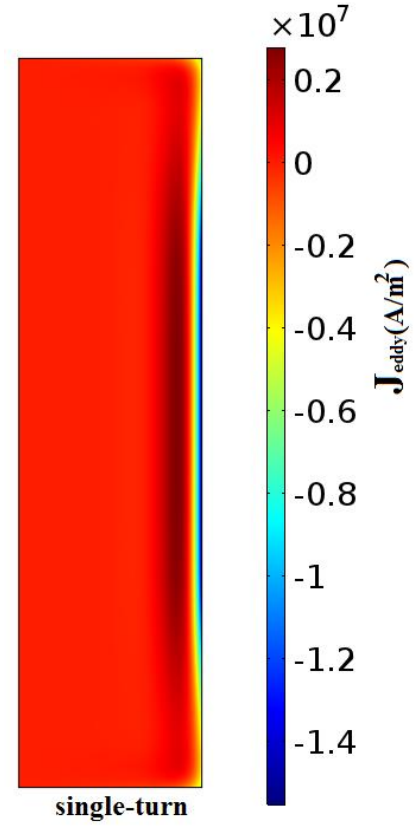

(a)

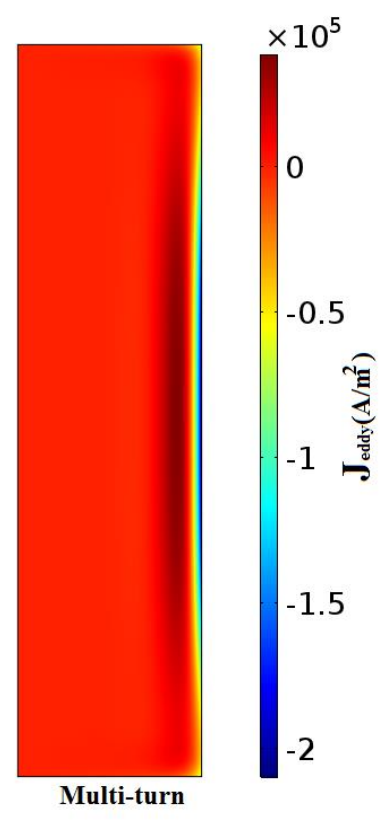

(b)

Figure 4. Intensity and distribution eddy currents density; (a) Single-turn coil and (b) Multi-turn coil 


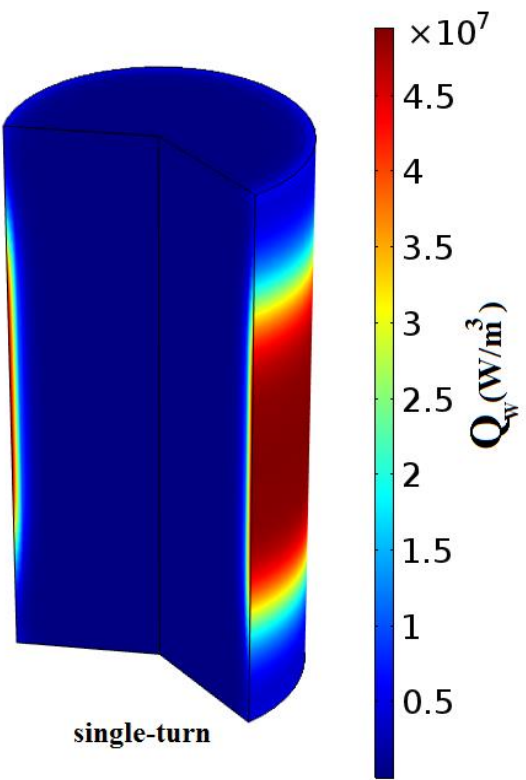

(a)

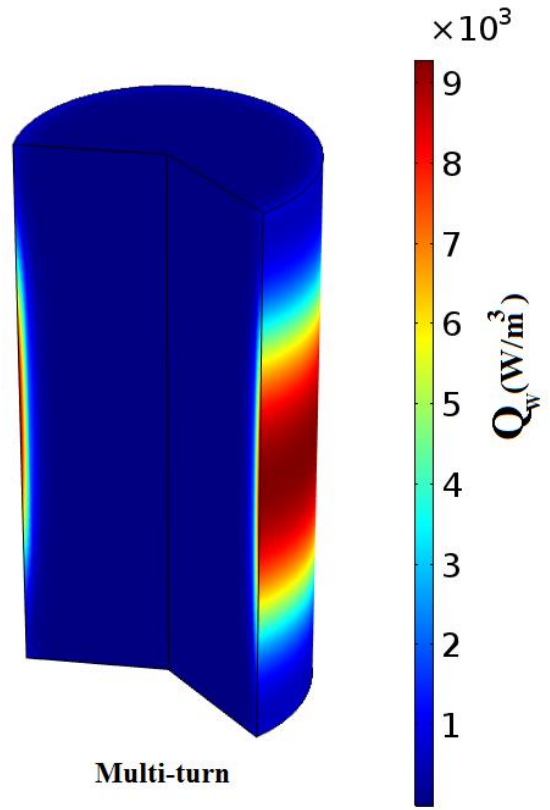

(b)

Figure 5. Intensity and distribution heat generated on the workpiece for; (a) Single-turn coil and (b) Multi-turn coil

In the Fig.6 the profile of generated heat in the Z-direction on the workpiece surface has been plotted. According to it, one can be found that in presence of Single-turn coil, maximum of generated heat on the workpiece covers more area than the Multi-turn coil. So procedure of changing of generation heat in the workpiece medium region is uniform in presence of Single-turn coil. While the change of the generated heat in the middle surface of the workpiece for Multi-turn coil is so faster.

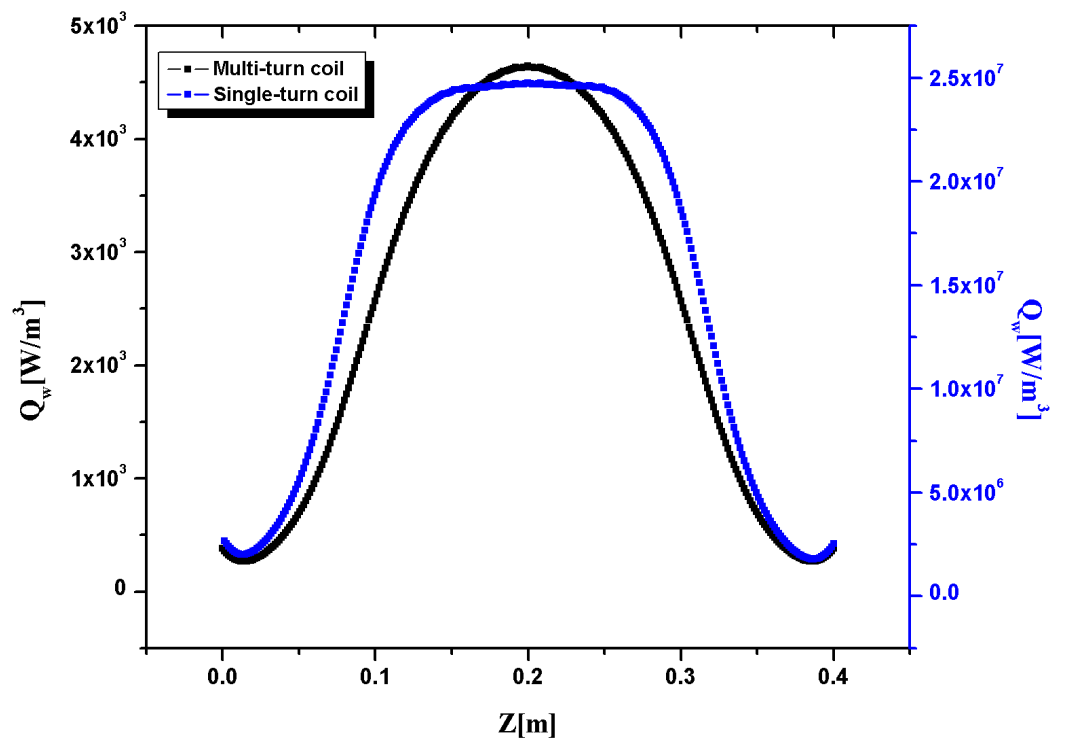

Figure 6. profile of distribution heat generated on the workpiece in direction Z-axis for; (a) Single-turn coil and (b) Multi-turn coil 


\section{Conclution}

This paper a procedure for the solution of induction heating coupled electromagnetic and thermal problems is presented. We has been analyzed the single-turn and Multi-turn coils in the induction heating process. The problem was considered as 2D and it has been solved using finite element method and COMSOL MULTIPHYSICS software. The results show that considering Single-turn and Multi-turn coils, as one of the parts important of induction heating system has significant effect on the electromagnetic field, eddy currents density, and generated heat distributions.

\section{References}

[1] G. M. Segura, "Induction heating converter's design, control and modelingapplied to continuous wire heating," Thesis, University Polittecnica of Catalunya (2012..

[2] V. Rudnev, D. Loveles, R. Cook, M. Black, "Handbook of Induction Heating," New York, 2003.

[3] P. Apostolidis, "Experimental and Numerical Investigation of Induction Heating in Asphalt Mixes," Thesis, Faculty Civil Engineering, Delft University of Technology, (2015).

[4] M. H. Tavakoli, H. Karbaschi, F. Samavat, "Influence of workpiece height on the induction heating process," Mathematical and Computer Modelling 54 (2011) 50-58.

[5] M.H. Tavakoli, "Modeling of Induction Heating in Oxide CzochralskiSystemsAdvantages and Problems," Crystal Growth \& Design (2008) , pp483-488.

[6] M.H. Tavakoli, A. Ojaghi, E. Mohammadi-Manesh, M. Mansour,"Influence of coil geometry on the induction heating process in crystal growth systems," J. Cryst. Growth , (2009) 1594-1599.

[7] https://www.comsol.com/ (AC/DC Module Users Guide).

[8] X. Zhou, B. G. Thomas, C. A. Hernandez, A. H.Castillejos,F. A. Acosta,"Measuring heat transfer during spray cooling using controlledinduction-heating experiments and computational models," Applied Mathematical Modelling 37 (2013) 3181-3192.

[9] Francesco Rui, "Numerical Analysis of Inhomogeneous Inductive Heating of Forging Parts," Thesis, University Hannover,(2015).

[10] C. XXIII, "Auto-Adaptive Multi-coil Inductor for Transverse Flux Induction Heating of Metal Strips," Thesis, University of Padova ,2012. 